\title{
On the Definiteness of Gauss-Kronrod Integration Rules
}

\author{
By Philip Rabinowitz
}

\begin{abstract}
The nondefiniteness of the Kronrod extension of the Gauss-Gegenbauer integration rule with weight function $w(x ; \mu)=\left(1-x^{2}\right)^{\mu-1 / 2}, 0<\mu<1$, is shown when there are more than three abscissas.
\end{abstract}

In a recent paper, Akrivis and Förster [1] have shown that the Clenshaw-Curtis and related integration rules are nondefinite, i.e., that the error $R f$ cannot be expressed in the form

$$
R f=c f^{(d+1)}(\xi)
$$

where $d$ is the precision of the rule. Using their approach combined with some of our previous results [3], we shall show that the same holds for the Kronrod extension (KE) of the Gauss-Gegenbauer integration rule (GGIR) with respect to the weight function

$$
w(x ; \mu)=\left(1-x^{2}\right)^{\mu-1 / 2}
$$

when $\mu$ satisfies $0<\mu<1$. In particular, the usual Gauss-Kronrod rule $(\mu=1 / 2)$ is nondefinite. We shall first give the results in [1] needed for our presentation. Then we shall introduce the KEGGIR. Finally, we shall prove the nondefiniteness of the KEGGIR for $\mu \in(0,1)$. The results on the KEGGIR appear in [3] and we shall not mention this in the sequel.

Consider the open integration rule $Q_{n}$ satisfying

$$
\int_{-1}^{1} w(x) f(x) d x=Q_{n} f+R_{n} f
$$

where

$$
Q_{n} f=\sum_{i=1}^{n} w_{i} f\left(x_{i}\right), \quad-1<x_{1}<x_{2}<\cdots<x_{n}<1,
$$

and $w(x)$ is a weight function which is positive for $x \in(-1,1) . Q_{n}$ is said to be of (exact) precision $d$ if $R_{n} f=0$ when $f$ is a polynomial of degree $\leqslant d$ and if there exists at least one polynomial $p$ of degree $d+1$ for which $R_{n} p \neq 0$. A slight generalization of Proposition 1 in [1] states that if there exists a function $f \in C[-1,1]$ such that $f^{(d+1)} \geqslant 0, f^{(d+1)} \equiv 0$ and $R_{n} f<0$, then the open rule $Q_{n}$ of precision $d$

Received February 11, 1985.

1980 Mathematics Subject Classification. Primary 65D30.

Key words and phrases. Definite integration rule, Kronrod rule, Gauss integration rule, Gegenbauer polynomials, Szegö polynomials. 
is nondefinite. We shall now introduce the KEGGIR, $Q_{2 n+1}$, and determine a function $f_{k}$ satisfying the hypotheses of this generalization. This will prove our claim that the KEGGIR's are nondefinite.

The abscissas $x_{i}, i=1, \ldots, n$, of the GGIR are the zeros of the Gegenbauer polynomial $C_{n}^{\mu}(x)$ and lie in $(-1,1)$. These polynomials are orthogonal with respect to $w(x ; \mu)$ and have the following normalization:

$$
\int_{-1}^{1} w(x ; \mu) C_{n}^{\mu}(x) C_{m}^{\mu}(x) d x=\delta_{n m} h_{n \mu}
$$

where

$$
h_{n \mu}=\pi^{1 / 2} \Gamma(n+2 \mu) \Gamma(\mu+1 / 2) /((n+\mu) n ! \Gamma(\mu) \Gamma(2 \mu)) .
$$

The KEGGIR, $Q_{2 n+1}$, is given by

$$
Q_{2 n+1} f=\sum_{i=1}^{n} u_{i} f\left(x_{i}\right)+\sum_{i=1}^{n+1} v_{i} f\left(y_{i}\right),
$$

where the $y_{i}$ are the zeros of the Szegö polynomial $E_{n+1, \mu}(x)$ which satisfies the orthogonality conditions

$$
\int_{-1}^{1} w(x ; \mu) C_{n}^{\mu}(x) E_{n+1, \mu}(x) x^{k} d x=0, \quad k=0,1, \ldots, n .
$$

For $0<\mu<1$, the $y_{i}$ lie in $(-1,1)$ so that $Q_{2 n+1}$ is an open integration rule. The precision $d$ of $Q_{2 n+1}$ is given by

$$
d= \begin{cases}3 n+1, & n \text { even }, \\ 3 n+2, & n \text { odd },\end{cases}
$$

for $0<\mu \leqslant 2, \mu \neq 1$. The Szegö polynomials are given by

$$
E_{n+1, \mu}(x)=\sum_{i=0}^{m-1} \lambda_{i \mu} T_{n+1-2 i}(x)+ \begin{cases}\lambda_{m \mu} T_{1}(x), & n \text { even, } \\ \frac{1}{2} \lambda_{m \mu}, & n \text { odd, }\end{cases}
$$

where $m=[(n+1) / 2]$ and the $T_{k}(x)$ are the Chebyshev polynomials of the first kind. The $\lambda_{i \mu}$ are given by

$$
\lambda_{0 \mu}=2 \gamma_{n \mu}^{-1}, \quad \sum_{i=1}^{k} f_{i, \mu} \lambda_{k-i, \mu}=0, \quad k=1,2, \ldots,
$$

where

$$
\begin{gathered}
\gamma_{n \mu}=\sqrt{\pi} \Gamma(n+2 \mu) / \Gamma(n+\mu+1), \quad f_{0 \mu}=1, \\
f_{j \mu}=(1-\mu / j)(1-\mu /(n+\mu+j)) f_{j-1, \mu},
\end{gathered}
$$

and we have not shown the dependence on $n$ of the $f_{j \mu}$ and the $\lambda_{i \mu}$. For $0<\mu<1$, the sequence $\left\{\lambda_{i \mu}: i=1,2, \ldots\right\}$ is strictly monotonic increasing.*

${ }^{*}$ Professor H. Brass has pointed out a gap in the proof in [3, p. 1279] that the sequence $\left\{\lambda_{i \mu}=\lambda_{i 0} \alpha_{i \mu}\right.$ : $i=1,2, \ldots\}$ is strictly monotonic increasing, since it does not follow that if a sequence $\left\{f_{j}: j=0,1, \ldots\right\}$ is strictly completely monotonic and $\sum_{i=0}^{\infty} \alpha_{i} u^{i}=\left\{\sum_{j=0}^{\infty} f_{j} u^{j}\right\}^{-1}$, then the sequence $\left\{-\alpha_{i}: i=1,2, \ldots\right\}$ is strictly completely monotonic. All that we can say is that it is completely monotonic. The following sequence provides a counterexample: $f_{j}=2^{-j}, \alpha_{0}=1, \alpha_{1}=-1 / 2, \alpha_{i}=0, i>1$. Professor Brass has also shown how to close this gap in our case, since if we did not have strict monotonicity, then $\lambda_{i \mu}=\lambda_{i+1, \mu}$ for some integer $i=i_{0}$. Hence by complete monotonicity, $\lambda_{i \mu}=\lambda_{i+1, \mu}$ for all $i \geqslant i_{0}$ which would imply that $F(u)=\sum_{j=0}^{\infty} f_{j \mu} u^{j}$ is a rational function. However, this is not the case since $F(u)$ is the hypergeometric function ${ }_{2} F_{1}(1-\mu, n+1 ; n+\mu+1 ; u)$ and $0<\mu<1$. 
The Gegenbauer polynomials and the Szegö polynomials are related by the following equality:

$$
C_{n}^{\mu}(x) E_{n+1, \mu}(x)=\sum_{i=0}^{n} c_{i} C_{n+1+i}^{\mu}(x),
$$

where the $c_{i}=c_{i}(\mu, n)$ are certain constants. For our purpose, the values of $c_{0}$ and $c_{1}$ are important. They are given by

$$
\begin{gathered}
c_{0}= \begin{cases}\frac{\gamma_{n \mu}}{2 \gamma_{n+1, \mu}}\left(\lambda_{m \mu}-\lambda_{m+1, \mu}\right), & n \text { even, } \\
0, & n \text { odd },\end{cases} \\
c_{1}= \begin{cases}\frac{\gamma_{n \mu}}{2 \gamma_{n+2, \mu}}\left(\lambda_{m-1, \mu}-\lambda_{m+1, \mu}\right), & n \text { odd }, \\
0, & n \text { even } .\end{cases}
\end{gathered}
$$

By the monotonicity of the sequence $\left\{\lambda_{i \mu}\right\}$, it follows that for $0<\mu<1, c_{0}$ is negative for $m \geqslant 1$, i.e., for $n \geqslant 2$ and $c_{1}$, for $m \geqslant 2$, i.e., for $n \geqslant 3$. We now define

$$
f_{k}(x)=C_{n}^{\mu}(x) E_{n+1, \mu}(x) C_{n+1+k}^{\mu}(x), \quad k=0,1 .
$$

Then, since $Q_{2 n+1} f_{k}=0$,

$$
R_{2 n+1} f_{k}=\int_{-1}^{1} w(x ; \mu) f_{k}(x) d x=c_{k} h_{n+1+k, \mu}, \quad k=0,1 .
$$

Furthermore, $f_{k}^{(3 n+2+k)}>0$. If $n$ is even, $c_{0} \neq 0$ and we choose $k=0$ so that $3 n+2+k=d+1$. If $n$ is odd, $c_{0}=0$ but $c_{1} \neq 0$ and we choose $k=1$ so that again $3 n+2+k=d+1$. In either case, $R_{2 n+1} f_{k}<0$ for $n \geqslant 2$ which implies that $Q_{2 n+1}$ is nondefinite. For $n=1, Q_{2 n+1}$ is the 3-point GGIR, which is definite.

For $\mu=0, Q_{2 n+1}$ is a Lobatto-Chebyshev rule of the first kind [2, p. 104] and hence is definite. Similarly, for $\mu=1, Q_{2 n+1}$ is a Gauss-Chebyshev rule of the second kind [2, p. 98], which is also definite. For $1<\mu \leqslant 2$, in which range KEGGIR's exist, the question of definiteness is still not settled. The same holds for the KE of the Lobatto-Gegenbauer integation rules except for that of the LobattoChebyshev rule of the first kind, which is itself a Lobatto-Chebyshev rule and hence is definite.

\section{Department of Applied Mathematics \\ The Weizmann Institute of Science \\ Rehovot 76100, Israel}

1. G. Akrivis \& K.-J. FOrSter, "On the definiteness of quadrature formulae of Clenshaw-Curtis type," Computing, v. 33, 1984, pp. 363-366.

2. P. J. Davis \& P. Rabinowitz, Methods of Numerical Integration, 2nd ed., Academic Press, New York, 1984.

3. P. Rabinowitz, "The exact degree of precision of generalized Gauss-Kronrod integration rules," Math. Comp., v. 35, 1980, pp. 1275-1283. 